\title{
Kinematic Modeling of the Main Solar Cycle
}

\author{
Vladimir Kaftan ${ }^{1}$ \\ ${ }^{1}$ Central Research Institute of Geodesy, Aerial Surveying and Cartography, Federal Agency of \\ Geodesy and Cartography, Moscow, Russia. kaftan@geod.ru
}

In spite of the great progress of solar physics research, complete understanding of the solar activity nature is still lacking. Although everybody can observe the obvious cyclical sunspot variation, recent models of the process can not describe it quite accurately. Thus the official forecast of the 23-rd solar cycle, mainly basing on the dynamical models and precursors, was not proved. Many official predictions considered that the cycle 23 will have an amplitude higher than the previous one. In this case statistical models were neglected as the less reliable but some of those kinematic forecasts are proved to this time. This situation makes evidence that we have to not ignore statistical approaches in the study of the solar behavior at the current stage of the knowledge.

We used three data sets of the solar activity characteristics as follows.

(a) International monthly sunspot numbers were taken from the SIDC web site of the Royal Observatory of Belgium.

(b) Solar radio flux $10.7 \mathrm{~cm}$ data were downloaded from the ISES Regional Warning Centre for Canada web site.

(c) Monthly sunspot areas (Greenwich general system) were taken from the Pulkovo Observatory database of the extended time series of solar activity indices (Nagovitsyn 1997).

The periodicity analysis technique named the sequential analysis of dominated harmonics, based on the iteration least squares method was used for mathematical modeling. The method allows to determine the trend component and the set of harmonics. The final model is a polyperiodical function that has the best fitting to the initial time series. Two steps of computations were used: 1) estimation and removal of the trend (linear component), 2) finding one after another and removal of the high amplitude periodical components (harmonics), twice exceeding their standard errors. Using the abovesaid method we have analyzed the time series of the monthly mean values of the sunspot indexes, sunspot areas and solar flux $10.7 \mathrm{~cm}$ units. Data sources were mentioned above. Kinematic models consisting of the trends and sets of dominated harmonics were constructed and extrapolations to 2010 were made for each characteristic. We have used the only the last $\sim 50$ year parts of the total series taking into account that the more modern data provide the more accurate short term forecast. This conclusion follows from the comparison of the latest author predictions well verified today (Demianov, Kaftan and Zubinsky 1999). The previous study (Demianov et al. 1999) shows that the kinematic forecasts of the current 23rd solar cycle come true pretty well. The present research allows us to predict the final stage of cycle 23 and the beginning of the next cycle 24 . The our new results have given a sufficient evidence that cycle 23 will be longer than usual in a contrast to many current official forecasts showing that the minimum will occur near 2006. Three different data models give us the assumption that the minimum epoch will take place between 2008 and 2009. The studies we have performed have demonstrated that the unusual local maximum will possibly be in 2005-2006. This bulge was demonstrated in the earlier prediction of the current cycle (Demianov et al. 1999). Now the 
probability of the event that cycle 24 will be a bit higher than the current one is reasonable but this presumption has to be studied thoroughly using the future observation results. For this reason we would like to provoke researchers to construct predictions of the next solar cycle and work on a collective solution using different techniques and methods. It is time to do that, because many of the recent predictions, for example (Joselyn et al. 1997, Kane 1997, Li 1997, Merzliakov 1997), were made earlier than 7 years ago. The past teaches us that solar cycle predictions based on kinematic approaches cannot be ignored as it was done in the last collective decision.

\section{References}

Demianov, G. V., Kaftan, V. I. and Zubinsky, V. I.: 1999, Participation of the Central Research Institute of Geodesy, Aerial Surveying and Cartography in the Third Baltic Sea Level GPS Campaign, Reports of the Finnish Geodetic Institute 99(4), 127-132.

SIDC web site of the Royal Observatory of Belgium: n.d. http://sidc.oma.be/index.php3.

ISES Regional Warning Centre for Canada web site: n.d. http://www.spaceweather.gc.ca.

Joselyn, J. A., Anderson, J. B., Coffey, H., Harvey, K., Hathaway, D., Heckman, G., Hildner, E., Mende, W., Schatten, K., Thompson, R., Thomson, A. W. P. and White, O. R.: 1997, Panel Achieves Consensus Prediction of Solar Cycle, 23. EOS, number 78, Trans. Amer. Geophys. Union, pp. 205,211-212.

Kane, R. P.: 1997, A Preliminary Estimate of the Size of the Coming Solar Cycle 23, Based on Ohl's Precursor Method, Geophysical Research Letters 24(15), 1899-1902.

Li, Y.: 1997, Predictions of the Features for Sunspot Cycle 23, Solar Physics 170, 437-445.

Merzliakov, V. I.: 1997, Solar Activity as a Result of Two-wave Magnetic Flux Generation, Solar Physics 170, 425-435.

Nagovitsyn, Y. A.: 1997, The Series of Total Sunspot Area Index in the Greenwich General System (1821-1989), Solnechnie Dannye, 1995-1996, russian edn, number 8, pp. 88-95. 